\title{
PERCOLATION ANALOG FOR A TWO-COMPONENT LIQUID-VAPOR SYSTEM
}

\author{
Joseph HOSHEN \\ Department of Chemistry, The Unversity of Michigan, Ann Arbor, Michigan 48I09, USA
}

Recerved 25 July 1979; in final form 25 July 1980

\begin{abstract}
Generalized site-bond percolation theory is utılized to provide an analog for a two-component liquid-vapor system in the phase transition region. The percolation model exhbits a behaviour typical of a non-ideal real hquid-vapor system with features such as separate curves for the hquid and vapor phases, and minumum and maxumum azeotrope formation.
\end{abstract}

The analogy between phase transition processes and percolation phenomena has been determuned in recent years. Kasteleyn and Fortuin [1] have shown that the bond percolation model is a special case of the AshkinTeller [2] and Potts [3] model for S-state atoms; thus providing a theoretical foundation in utilizing the percolation model as an analog [4] for phase transition phenomena. Recently Stauffer [5] has emphasized the analogy between percolation, magnetization, hquidvapor transition and polymer gelation.

In this letter I would like to explore the analogy between the generalized site-bond problem [6] and a two-component hquid-vapor system. In thus system the finite clusters and the unfinte cluster provide the vapor and the condensed phase analogs [5], respectively. The percolation threshold is reached when finite clusters ("vapor") coalesce to form an infinite cluster ("liquid").

Let us now consider a percolation model for a binary muxture of A and B atoms, randomly distributed over a lattıce. Each lattice site is occupied with an $A$ atom or a $\mathrm{B}$ atom with a probability $C_{\mathrm{A}}$ or $C_{\mathrm{B}}$, respectively such that $C_{\mathrm{A}}+C_{\mathrm{B}}=1$. In a real liquid-vapor system $C_{\mathrm{A}}$ and $C_{\mathrm{B}}$ would denote the mole fraction of the $\mathrm{A}$ and $B$ components.

Kasteleyn and Fortuin's [1] prescription for the bond probabiluties can be extended to the generalized site-bond problem. Thus, one could wnte the following bond probabilities for the $A$ and $B$ atoms:

\footnotetext{
* Supported by NSF Grant DMR75-07832 A01.
}

$p_{\mathrm{AA}}=1-\exp \left(-J_{\mathrm{AA}} / T\right)$,
$p_{\mathrm{AB}}=1-\exp \left(-J_{\mathrm{AB}} / T\right)$,
$p_{\mathrm{BB}}=1-\exp \left(-J_{\mathrm{BB}} / T\right)$.

Eqs.(1)-(3) correspond to the bond probabilities between $\mathbf{A}-\mathbf{A}, \mathbf{A}-\mathbf{B}$ and $\mathbf{B}-\mathbf{B}$ atoms, respectively. $J_{\mathrm{AA}}, J_{\mathrm{AB}}$ and $J_{\mathrm{BB}}$ are the corresponding interaction parameters and $T$ is the temperature given in arbitrary units. There are several interesting limiting values for eqs. (1)-(3). Settıng $\boldsymbol{J}_{\mathrm{AB}}=\boldsymbol{J}_{\mathrm{BB}}=\mathbf{0}$, we get in the limit as $T \rightarrow 0$ that $p_{\mathrm{AA}} \rightarrow 1$ and $p_{\mathrm{AB}}=p_{\mathrm{BB}}=0$ which correspond to the pure site problem.

In the pure site (or pure bond) case the theshold value $C^{*}$ defines a single point on the $C$ axis for which the percolation probability $p(C)=0$. For all values $C>C^{*}, p(C)>0$. In our site-bond case $p$ is defined by two independent variables $C_{\mathrm{A}}$ and $T$; thus, the percolation transition is defined by a curve $p\left(C_{\mathrm{A}}, T\right)=0$ which we named the critical percolation curve [6].

In an infinte percolating system the fraction of the infinite cluster, given by $p$, is a continuous function, whereas at the critical points only the derivatives of $p$ are not continuous, implying that the percolation transition is second order. On the transition curve the composition of the "vapor" coincides with the compositron of the entire sample as the infinite cluster is present in infinitesumal amount in the system. On the other hand, the infinite cluster deviates from the system composition. For a finite $p$, both the "liquid" and "vapor" of the percolating system deviate from the overall sample composition. 
The binary percolation system is investigated by utilizing the Monte Carlo method. A simple cubic crystal (with cyclic boundary conditions) is simulated on the computer. A and B atoms are generated at random on the lattice sites. The bond formation for every two nearest-neighbor atoms is determined by eqs. (1) -. (3), The system is then analyzed by utilizing the extended [6] ciuster multiple labeling technique [7] (ECMLT). This method enables us to determine the number of clusters and their sizes in the simulated lattice.

Simulating for a given set $J_{\mathrm{AA}}, J_{\mathrm{AB}}$, and $J_{\mathrm{BB}}$, one starts at some $C_{\mathrm{A}}$ value and a temperature $T$, high enough, so that only small clusters are encountered in the system. The system is then "cooled" until small clusters condense to form a large percolating cluster ${ }^{\neq}$ at some threshold temperature $T_{\mathrm{c}}$. At the threshold temperature the number of the $\mathrm{A}$ atoms is determined for the large percolating cluster as well as for the remaining small clusters. Invoking the analogy between percolation and the liquid-vapor system, we can now determine the concentration of $\mathrm{A}$ atoms in the "liquid" and "vapor" phases of the percolation model. Repeating the computer experiment for several values of $C_{\mathrm{A}}$ (and fixed $J$ ) we can determine the "liquid-vapor" curves for the percolation model.

The results of the Monte Carlo simulation for three sets of nearest-neighbor interactions $J_{\mathrm{AA}}, J_{\mathrm{AB}}$ and $J_{\mathrm{BB}}$ for simple cubic lattices containing $30^{3}$ atoms are presented in figs. 1 and 2 . Some interesting observations for these curves can be made: (a) The "liquid" and "vapor" curves do not coincide for a given interaction parameter set (except when $J_{\mathrm{AA}}=J_{\mathrm{AB}}=J_{\mathrm{BB}}$ ). (b) An azeotrope is formed at some concentration value $C_{A}$ for $J_{A B}>J_{A A}$ and $J_{A B}>J_{B B}$, or $J_{A B}<J_{A A}$ and $J_{A B}<J_{B B}$. (c) The "vapor" eurve for the A component is essentially linear with the concentration for $J_{\mathrm{AB}}=\left(J_{\mathrm{AA}} J_{\mathrm{BB}}\right)^{1 / 2}$. In this case no azeotrope is formed (see fig. 1).

It should be noted that in our computer experiment the critical percolation curve (dashed line) which represents the entire system composition does not coincide with the sample "vapor" curve. This could be attributed to the finite size of our sample. We expect this deviation to decrease as we increase our sample

\footnotetext{
* Note that in the simulation we investigate a finite system for which we have to define an "infinite" percolating cluster. A practical solution to this problem is described in ref. [7].
}

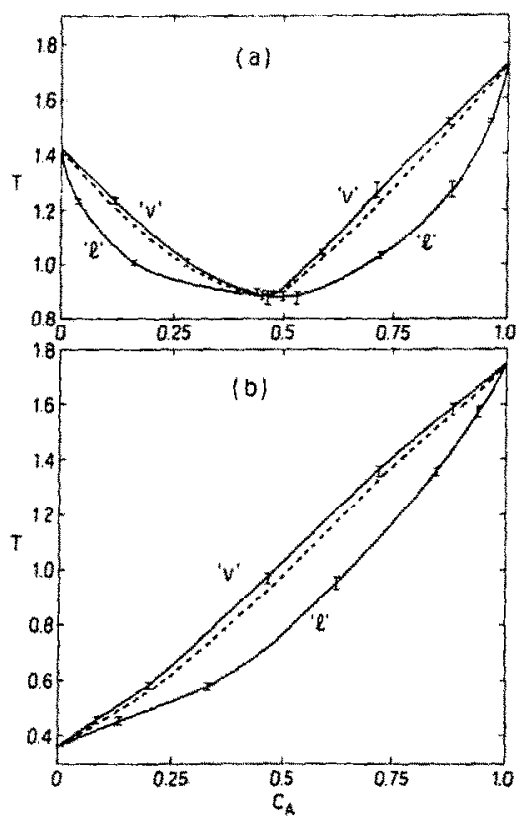

Fig. 1. Percolation analog for a binary mixture (lattice size 30 $\times 30 \times 30$ ). (a) $J_{\mathrm{AA}}=0.5, J_{\mathrm{AB}}=0.1, J_{\mathrm{BB}}=0.4$. "v" curve denotes small cluster composition ("vapor") whereas " $\mathrm{l}$ " donotes the percolating cluster composition ("liquid"). An ateotrope is formed at $C_{\mathrm{A}}=0.45$. The temperature $T$ and the interactions $J$ are given in arbitrary units. (b) $J_{\mathrm{AA}}=0.5, J_{\mathrm{BB}}=0.1$, $J_{\mathrm{AB}}=\left(J_{\mathrm{AA}} J_{\mathrm{BB}}\right)^{1 / 2}$. The dashed line denotes the critical percolation curve [6]. As expected, no azeotrope is formed for this case. The linear behavior of the " $\mathrm{"} \mathrm{curve} \mathrm{is} \mathrm{possibly} \mathrm{due}$ to the geometric mean choice of $J_{\mathrm{AB}}$.

sizes. We hope also in the future to present data on some of the critical exponents pertaining to our "liquid-vapor" system.

At first glance the percolation model presented in this letter seems to have a rather close resemblance to the lattice gas model. However, it can be shown that the two models are distinct. This distinction can be best illustrated through the formalism presented in the Kasteleyn-Fortuin [1] general cluster theory. In this theory [1] the bond percolation problem corresponds to a single-state atom whereas the Ising model, which can be translated formally to the lattice gas models, is based on two states per atom [8]. Hence the number of possible lattice states is 1 and $2^{n}$ for the percolation 


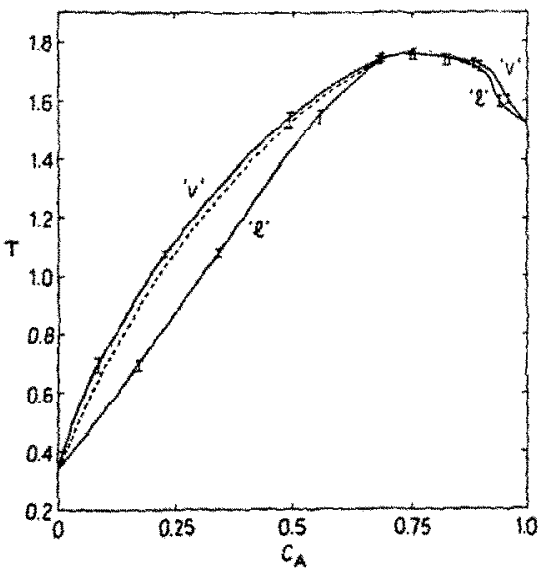

Fig. 2. Percolation analog for a binary mixture (lattice size $30 \times 30 \times 30) . J_{\mathrm{AA}}=0.45, J_{\mathrm{AB}}=0.6, J_{\mathrm{BB}}=0.1$. An azeotrope is formed at $C_{A}=0.75$. It should be noted that for $c_{\mathrm{A}}>0.7$ the critical percolation curve (dashed line) coincides with the " $\mathrm{y}$ " curve.)

and the Ising models, respectively, at a given lattice configuration where $n$ is the number of atoms in the lattice. Under these circumstances, there cannot be variation in time for the percolation model. Monte Carlo time evolution simulations $[8-10]$ which proved to be useful for the lattice gas model, are meaningless in the framework of the percolation model. Metastable states and supersaturation in finite systems [9] cannot be described within the framework of the percolation model. It should also be noted that the Kasteleyn-
Fortuin cluster model [1] can be related to classical thermodynamics which has been very useful in describing, among others, the macroscopic properties of binary liquid-vapor systems [11].

The author would like to thank Professor $\mathbf{R}$. Kopelman for very helpful discussions of the topic and Professor T.M. Dunn for his suggestions and comments. The author would like also to thank Professors D. Staufier and $W$. Klein for their valuable suggestions and comments on the critical aspects of the transition.

\section{References}

(1) P.W. Kasteleyn and C.M. Fortuin, J. Phys, Soc, Japan Suppl. $26(1269) 11$.

[2] J. Ashkin and E. Teller, Phys. Rev, 64 (1943) 178 ,

(3) R.B. Potts, Proc, Cambridge Phil. Soc. 48 (1952) 106.

14) S. Kitkpatrick, Phys, Rev. Letters 36 (1976) 69.

[5] D. Stauffer, J. Chem. Soc. Naraday Trans. II 72 (1976) 1354.

[6] J. Hoshen, P. Klymko and R. Kopelman, J. Stat. Phys., to be publisined.

(7) J. Hoshen and R. Kopelman, Phys. Rev. B14 (1976) 3438.

[8] H. Muller-Krumbhaar and E.P. Stoll, J. Chem. Phys. 65 (1976) 4294

[9] A.B. Bortz, M.H. Kalos, J.L. Lebowitz and M.A. Zendejas, Phys, Rev, B10 (1974) 535.

[10] M. Rao, B.J. Berne and M.H. Kalos, J. Chem. Phys. 68 (1978) 1325

[11] E.A. Guggenheim, Mixtures (Oxford Univ. Press, London, 1952) p. 32. 